\title{
Representation of Multivariate Functions via the Potential Theory and Applications to Inequalities
}

\author{
Florica C. Cîrstea ${ }^{1}$ and Sever S. Dragomir ${ }^{2}$ \\ ${ }^{1}$ Department of Mathematics, Australian National University, Canberra, ACT 0200, Australia \\ ${ }^{2}$ School of Computer Science and Mathematics, Victoria University, P.O. Box 14428, Melbourne City, \\ Victoria 8001, Australia
}

Correspondence should be addressed to Sever S. Dragomir, sever.dragomir@vu.edu.au

Received 12 February 2007; Revised 2 August 2007; Accepted 9 November 2007

Recommended by Siegfried Carl

We use the potential theory to give integral representations of functions in the Sobolev spaces $W^{1, p}(\Omega)$, where $p \geq 1$ and $\Omega$ is a smooth bounded domain in $\mathbb{R}^{N}(N \geq 2)$. As a byproduct, we obtain sharp inequalities of Ostrowski type.

Copyright (C) 2008 F. C. Cîrstea and S. S. Dragomir. This is an open access article distributed under the Creative Commons Attribution License, which permits unrestricted use, distribution, and reproduction in any medium, provided the original work is properly cited.

\section{Introduction and main results}

Let $N \geq 2$ and let $\langle\cdot, \cdot\rangle$ denote the canonical inner product on $\mathbb{R}^{N} \times \mathbb{R}^{N}$. If $\omega_{N}$ stands for the area of the surface of the $(N-1)$-dimensional unit sphere, then $\omega_{N}=2 \pi^{N / 2} / \Gamma(N / 2)$, where $\Gamma$ is the gamma function defined by $\Gamma(s)=\int_{0}^{\infty} e^{-t} t^{s-1} d t$ for $s>0$ (see [1, Proposition 0.7]).

Let $E$ denote the normalized fundamental solution of Laplace equation:

$$
E(x)= \begin{cases}\frac{1}{2 \pi} \ln |x|, & x \neq 0 \text { if } N=2, \\ \frac{1}{(2-N) \omega_{N}|x|^{N-2},} & x \neq 0 \text { if } N \geq 3 .\end{cases}
$$

Unless otherwise stated, we assume throughout that $\Omega \subset \mathbb{R}^{N}$ is a bounded domain with $C^{2}$ boundary $\partial \Omega$. Let $v$ denote the unit outward normal to $\partial \Omega$ and let $d \sigma$ indicate the $(N-1)$-dimensional area element in $\partial \Omega$. The Green-Riemann formula says that any function 
$f \in C^{2}(\Omega) \cap C^{1}(\bar{\Omega})$ satisfying $\Delta f \in C(\bar{\Omega})$ can be represented in $\Omega$ as follows (see [2, Section $2.4]):$

$$
f(y)=\int_{\partial \Omega}\left(f(x) \frac{\partial E}{\partial v}(x-y)-\frac{\partial f}{\partial \mathcal{v}}(x) E(x-y)\right) d \sigma(x)+\int_{\Omega} E(x-y) \Delta f(x) d x, \quad \forall y \in \Omega,
$$

where $(\partial f / \partial v)(x)$ is the normal derivative of $f$ at $x \in \partial \Omega$. In particular, if $f \in C_{0}^{\infty}(\Omega)$ (the set of functions in $C^{\infty}(\Omega)$ with compact support in $\Omega$ ), then (1.2) leads to the representation formula

$$
f(y)=\int_{\Omega} E(x-y) \Delta f(x) d x, \quad \forall y \in \Omega
$$

For a continuous function $h$ on $\partial \Omega$, the double-layer potential with moment $h$ is defined by

$$
\overline{\bar{u}}_{h}(y)=\int_{\partial \Omega} h(x) \frac{\partial E}{\partial v}(x-y) d \sigma(x)
$$

Expression (1.4) may be interpreted as the potential produced by dipoles located on $\partial \Omega$; the direction of which at any point $x \in \partial \Omega$ coincides with that of the exterior normal $v$, while its intensity is equal to $h(x)$. The double-layer potential is well defined in $\mathbb{R}^{N}$ and it satisfies the Laplace equation $\Delta u=0$ in $\mathbb{R}^{N} \backslash \partial \Omega$ (see Proposition 2.8). For other properties of the double-layer potential, see Lemma 2.9 and Proposition 2.10.

The double-layer potential plays an important role in solving boundary value problems of elliptic equations. The representation of the solution of the interior/exterior Dirichlet problem for Laplace's equation is sought as a double-layer potential with unknown density $h$. An application of property (2.14) leads to a Fredholm equation of the second kind on $\partial \Omega$ in order to determine the function $h$ (see, e.g., [3]).

In many problems of mathematical physics and variational calculus, it is not sufficient to deal with classical solutions of differential equations. One needs to introduce the notion of weak derivatives and to work in Sobolev spaces, which have become an indispensable tool in the study of partial differential equations.

For $1 \leq p \leq \infty$, we denote by $W^{1, p}(\Omega)$ the Sobolev space defined by

$$
W^{1, p}(\Omega)=\left\{u \in L^{p}(\Omega) \mid \begin{array}{c}
\exists g_{1}, g_{2}, \ldots, g_{N} \in L^{p}(\Omega) \text { such that } \\
\int_{\Omega} u \frac{\partial \phi}{\partial x_{i}} d x=-\int_{\Omega} g_{i} \phi d x, \forall \phi \in C_{0}^{\infty}(\Omega), \forall i \in\{1,2, \ldots, N\}
\end{array}\right\} .
$$

For $u \in W^{1, p}(\Omega)$, we define $g_{i}=\partial u / \partial x_{i}$ and write $\nabla u=\left(\partial u / \partial x_{1}, \partial u / \partial x_{2}, \ldots, \partial u / \partial x_{N}\right)$. The Sobolev space $W^{1, p}(\Omega)$ is endowed with the norm

$$
\|u\|_{W^{1, p}(\Omega)}=\|u\|_{L^{p}(\Omega)}+\sum_{i=1}^{N}\left\|\frac{\partial u}{\partial x_{i}}\right\|_{L^{p}(\Omega)},
$$

where $\|\cdot\|_{L^{p}(\Omega)}$ stands for the usual norm on $L^{p}(\Omega)$. The closure of $C_{0}^{\infty}(\Omega)$ in the norm of $W^{1, p}(\Omega)$ is denoted by $W_{0}^{1, p}(\Omega)$. For details on Sobolev spaces, we refer to [2,4], or [5]. 
Since $\Omega$ is bounded, we have $C^{1}(\bar{\Omega}) \subset W^{1, \infty}(\Omega) \subseteq W^{1, p}(\Omega)$ for every $p \in[1, \infty]$. 2.3):

The following representation holds for functions $f$ in $W_{0}^{1, p}(\Omega)$ with $p \geq 1$ (see Remark

$$
f(y)=-\int_{\Omega}\langle\nabla E(x-y), \nabla f(x)\rangle d x \quad \text { a.e. } y \in \Omega \text {. }
$$

We first give an integral representation of functions in $W^{1, p}(\Omega)$ for any $p \geq 1$.

Theorem 1.1. For any $g \in W^{1, p}(\Omega)$ with $p \geq 1$, there is a sequence $\left(g_{n}\right)$ in $C^{\infty}(\bar{\Omega})$ such that

$$
\begin{aligned}
& g(y)=\lim _{n \rightarrow \infty} \int_{\partial \Omega} g_{n}(x) \frac{\partial E}{\partial v}(x-y) d \sigma(x)-\int_{\Omega}\langle\nabla E(x-y), \nabla g(x)\rangle d x \quad \text { a.e. } y \in \Omega, \\
& 0=\lim _{n \rightarrow \infty} \int_{\partial \Omega} g_{n}(x) \frac{\partial E}{\partial v}(x-y) d \sigma(x)-\int_{\Omega}\langle\nabla E(x-y), \nabla g(x)\rangle d x, \quad \forall y \in \mathbb{R}^{N} \backslash \bar{\Omega} .
\end{aligned}
$$

Remark 1.2. If $g \in W_{0}^{1, p}(\Omega)$, then there exists a sequence $\left(g_{n}\right)$ in $C_{0}^{\infty}(\Omega)$ for which (1.8) holds. Thus, we regain (1.7) for any function $f$ in $W_{0}^{1, p}(\Omega)$.

Under a suitable smoothness condition, the representation of Theorem 1.1 can be refined for functions in $W^{1, p}(\Omega)$ with $p>N$ (see Theorem 1.3). Using Morrey's inequality, one can prove that functions in the Sobolev space $W^{1, p}(\Omega)$ with $p>N$ are classically differentiable almost everywhere in $\Omega$ (cf. [2, page 176] or [4]). By Proposition 2.13, any function in $W^{1, p}(\Omega)$ with $N<p<\infty$ is uniformly Hölder continuous in $\Omega$ with exponent $1-N / p$ (after possibly being redefined on a set of measures 0$)$. In particular, any function in $W^{1, p}(\Omega)$ with $p>N$ is continuous on $\bar{\Omega}$, and thus it has a well-defined trace which is bounded.

The proof of Theorem 1.1 relies on the density of $C^{\infty}(\bar{\Omega})$ in $W^{1, p}(\Omega)$ as well as the following result.

Theorem 1.3. Assume that $f \in W^{1, p}(\Omega) \cap C^{1}(\Omega \backslash A)$, where $p \geq 1$ and $A=\left(a_{i}\right)_{i \in I}$ is a finite family of points in $\Omega$.

(a) If $p>N$, then $f$ can be represented as follows:

$$
f(y)= \begin{cases}\overline{\bar{u}}_{f}(y)-\int_{\Omega}\langle\nabla E(x-y), \nabla f(x)\rangle d x, & \forall y \in \Omega, \\ 2\left(\overline{\bar{u}}_{f}(y)-\int_{\Omega}\langle\nabla E(x-y), \nabla f(x)\rangle d x\right), & \forall y \in \partial \Omega .\end{cases}
$$

(b) If $p \geq 1$ and $f \in C(\bar{\Omega})$, then

$$
0=\overline{\bar{u}}_{f}(y)-\int_{\Omega}\langle\nabla E(x-y), \nabla f(x)\rangle d x, \quad \forall y \in \mathbb{R}^{N} \backslash \bar{\Omega}
$$


Remark 1.4. (i) If $f=1$ on $\bar{\Omega}$, then Theorem 1.3 recovers Gauss formula (see Lemma 2.9).

(ii) Theorem 1.3 leads to the mean value theorems for harmonic functions (see Remark 5.4).

(iii) If $f \in C^{2}(\Omega) \cap C^{1}(\bar{\Omega})$ such that $\Delta f \in C(\bar{\Omega})$, then by combining Theorem 1.3 and Proposition 2.7, we regain the Green-Riemann representation formula (1.2).

This paper is organized as follows. In Section 2, we include some known results that are necessary later in the paper. Section 3 is dedicated to the proof of Theorem 1.3. Based on it, we prove Theorem 1.1 in Section 4. We conclude the paper with a representation of smooth functions in $W^{1, p}(\Omega)$ with $p>N$ in terms of the integral mean value over the domain (see Theorem 5.1 in Section 5). As a byproduct of our main results, we obtain a sharp estimate of the difference between the value of a function $f$ and the double-layer potential with moment $f$.

\section{Preliminaries}

Lemma 2.1 (see [4, Theorem IV.9]). Let $\omega \subset \mathbb{R}^{N}$ be an open set. Let $\left(h_{n}\right)$ be a sequence in $L^{p}(\omega)$, $1 \leq p \leq \infty$, and let $h \in L^{p}(\omega)$ be such that $\left\|h_{n}-h\right\|_{L^{p}(\omega)} \rightarrow 0$.

Then, there exist a subsequence $\left(h_{n_{k}}\right)$ and a function $\varphi \in L^{p}(\omega)$ such that

(a) $h_{n_{k}}(x) \rightarrow h(x)$ a.e. in $\omega$,

(b) $\left|h_{n_{k}}(x)\right| \leq \varphi(x)$ for all $k$, a.e. in $\omega$.

For fixed $y \in \mathbb{R}^{N}$, we define the operator $\boldsymbol{\kappa}_{j}$ by

$$
\left(\mathcal{K}_{j} u\right)(y)=\int_{\Omega} \frac{x_{j}-y_{j}}{|x-y|^{N}} u(x) d x, \quad j \in\{1,2, \ldots, N\}
$$

Lemma 2.2. (i) If $1 \leq p \leq N$, then the operator $\mathcal{K}_{j}: L^{p}(\Omega) \rightarrow L^{p}(\Omega)$ is compact.

(ii) If $p>N$, then the operator $\mathcal{K}_{j}: L^{p}(\Omega) \rightarrow C(\bar{\Omega})$ is compact.

Remark 2.3. If $\Omega \subset \mathbb{R}^{N}$ is a bounded domain and $f \in W_{0}^{1, p}(\Omega)$ with $p \geq 1$, then (1.7) holds. Indeed, $E(x)$ given by (1.1) has weak derivatives and $\left(\partial / \partial x_{j}\right) E(x-y)=\left(1 / \omega_{n}\right)\left(\left(x_{j}-y_{j}\right) / \mid x-\right.$ $\left.\left.y\right|^{N}\right)$ for every $j \in\{1,2, \ldots, N\}$. If $f \in C_{0}^{\infty}(\Omega)$, then by the definition of weak derivatives, we have

$$
\int_{\Omega} E(x-y)(\Delta f)(x) d x=-\sum_{j=1}^{N} \int_{\Omega} \frac{\partial E(x-y)}{\partial x_{j}} \frac{\partial f}{\partial x_{j}} d x=-\int_{\Omega}\langle\nabla E(x-y), \nabla f(x)\rangle d x .
$$

Thus, using (1.3), we find (1.7) for every $y \in \Omega$. Now, if $f \in W_{0}^{1, p}(\Omega)$, we take a sequence $\left(f_{n}\right)_{n \geq 1}$ in $C_{0}^{\infty}(\Omega)$ such that $f_{n} \rightarrow f$ in $W^{1, p}(\Omega)$ as $n \rightarrow \infty$. Thus, for each $f_{n}$ with $n \geq 1$, we have

$$
f_{n}(y)=-\frac{1}{\omega_{N}} \sum_{j=1}^{N} \mathcal{K}_{j}\left(\frac{\partial f_{n}}{\partial x_{j}}\right)(y), \quad \forall y \in \Omega
$$


By Lemma 2.2, each operator $\mathcal{K}_{j}$ is compact from $L^{p}(\Omega)$ to $L^{p}(\Omega)$. Thus, $\partial f_{n} / \partial x_{j} \rightarrow \partial f / \partial x_{j}$ in $L^{p}(\Omega)$ as $n \rightarrow \infty$ implies that $\mathcal{K}_{j}\left(\partial f_{n} / \partial x_{j}\right) \rightarrow \mathcal{K}_{j}\left(\partial f / \partial x_{j}\right)$ in $L^{p}(\Omega)$ as $n \rightarrow \infty$. By Lemma 2.1, we have (up to a subsequence of $\left.\left(f_{n}\right)\right) \lim _{n \rightarrow \infty} \mathcal{K}_{j}\left(\partial f_{n} / \partial x_{j}\right)(y)=\mathcal{K}_{j}\left(\partial f / \partial x_{j}\right)(y)$ and $\lim _{n \rightarrow \infty} f_{n}(y)=f(y)$ a.e. $y \in \Omega\left(\right.$ since $f_{n} \rightarrow f$ in $L^{p}(\Omega)$ as $\left.n \rightarrow \infty\right)$. By passing to the limit in (2.3), we conclude (1.7).

Lemma 2.4 (see [5, Lemma 5.47]). Let $y \in \mathbb{R}^{N}$ and let $\omega$ be a domain of finite volume in $\mathbb{R}^{N}$. If $0 \leq r<N$, then

$$
\int_{\omega}|x-y|^{-\gamma} d x \leq K|\omega|^{1-\gamma / N}
$$

where the constant $K$ depends on $\gamma$ and $N$ but not on $y$ or $\omega$.

By a vector field, we understand an $\mathbb{R}^{N}$-valued function on a subset of $\mathbb{R}^{N}$. If $Z=$ $\left(z_{1}, z_{2}, \ldots, z_{N}\right)$ is a differentiable vector field on an open set $\omega \subset \mathbb{R}^{N}$, the divergence of $Z$ on $\omega$ is defined by

$$
\operatorname{div} Z=\sum_{i=1}^{N} \frac{\partial z_{i}}{\partial x_{i}}
$$

Proposition 2.5 (the divergence theorem). If $\omega \subset \mathbb{R}^{N}$ is a bounded domain with $C^{1}$ boundary and $Z$ is a vector field of class $C^{1}(\omega) \cap C(\bar{\omega})$, then

$$
\int_{\omega} \operatorname{div} Z(y) d y=\int_{\partial \omega}\langle Z(x), v(x)\rangle d \sigma(x) .
$$

If $\omega$ is a domain to which the divergence theorem applies, then we have the following.

Proposition 2.6 (Green's first identity). If $u, v \in C^{2}(\omega) \cap C^{1}(\bar{\omega})$, then the following holds:

$$
\int_{\omega} v(x) \Delta u(x) d x+\int_{\omega}\langle\nabla u(x), \nabla v(x)\rangle d x=\int_{\partial \omega} v(x) \frac{\partial u}{\partial v}(x) d \sigma(x) .
$$

Proposition 2.7. Let $\Omega$ be a bounded domain with $C^{1}$ boundary. If $f \in C^{2}(\Omega) \cap C^{1}(\bar{\Omega})$ such that $\Delta f \in C(\bar{\Omega})$, then for every $y \in \mathbb{R}^{N} \backslash \partial \Omega$, one has

$$
\int_{\Omega}\langle\nabla E(x-y), \nabla f(x)\rangle d x=\int_{\partial \Omega} \frac{\partial f}{\partial v}(x) E(x-y) d \sigma(x)-\int_{\Omega} E(x-y) \Delta f(x) d x .
$$

Proof. If $y \in \mathbb{R}^{N} \backslash \bar{\Omega}$, then (2.8) follows from Proposition 2.6 (since $x \mapsto E(x-y)$ belongs to $\left.C^{2}(\Omega) \cap C^{1}(\bar{\Omega})\right)$. For $y \in \Omega$ fixed, we choose $\epsilon>0$ such that $\bar{B}_{\epsilon}(y) \subset \Omega$, where $B_{\epsilon}(y)$ denotes the open ball of radius $\epsilon>0$ centered at $y$. By Proposition 2.6 (applied on $\Omega \backslash \bar{B}_{\epsilon}(y)$ ), we find

$$
\begin{aligned}
\int_{\Omega \backslash \bar{B}_{\epsilon}(y)} E(x-y) \Delta f(x) d x= & \int_{\partial \Omega} \frac{\partial f}{\partial \mathcal{v}}(x) E(x-y) d \sigma(x)-\int_{\partial B_{\epsilon}(y)} \frac{\partial f}{\partial \mathcal{v}}(x) E(x-y) d \sigma(x) \\
& -\int_{\Omega \backslash \bar{B}_{\epsilon}(y)}\langle\nabla f(x), \nabla E(x-y)\rangle d x .
\end{aligned}
$$


Since $\Delta f \in C(\bar{\Omega})$ and $f \in C^{1}(\bar{\Omega})$, we have that $x \mapsto E(x-y) \Delta f(x)$ and $x \mapsto \int_{\Omega}\langle\nabla E(x-$ $y), \nabla f(x)\rangle d x$ are integrable on $\Omega$. We see that

$$
I_{\epsilon}:=\int_{\partial B_{\epsilon}(y)} \frac{\partial f}{\partial \mathcal{v}}(x) E(x-y) d \sigma(x) \longrightarrow 0 \quad \text { as } \epsilon \longrightarrow 0 .
$$

Indeed, for some constant $C>0$, we have

$$
I_{\epsilon} \leq \begin{cases}\frac{1}{2 \pi} \int_{\partial B_{\epsilon}(y)}\left|\frac{\partial f}{\partial \mathcal{V}}(x) \ln \right| x-y|| d \sigma(x) \leq-C \epsilon \ln \epsilon & \text { if } N=2, \\ \frac{1}{\omega_{N}(N-2)} \int_{\partial B_{\epsilon}(y)}\left|\frac{\partial f}{\partial \mathcal{v}}(x)\right| \frac{d \sigma(x)}{|x-y|^{N-2} \leq C \omega_{N} \epsilon} \quad \text { if } N \geq 3 .\end{cases}
$$

Thus, passing to the limit $\epsilon \rightarrow 0$ in (2.9) and using (2.10), we obtain (2.8). $[1])$.

We next give some properties of the double-layer potential $\overline{\bar{u}}_{h}(y)$ defined by (1.4) (see

Proposition 2.8. If $h$ is a continuous function on $\partial \Omega$, then

(i) $\overline{\bar{u}}_{h}(y)$ given by (1.4) is well defined for all $y \in \mathbb{R}^{N}$,

(ii) $\Delta \overline{\bar{u}}_{h}(y)=0$ for all $y \in \mathbb{R}^{N} \backslash \partial \Omega$.

Lemma 2.9. Let $\overline{\bar{v}}$ be the double-layer potential with moment $h \equiv 1$, that is,

$$
\overline{\bar{v}}(y)=\int_{\partial \Omega} \frac{\partial E}{\partial v}(x-y) d \sigma(x)
$$

Then, one has

$$
\overline{\bar{v}}(y)= \begin{cases}1 & \text { if } y \in \Omega, \\ \frac{1}{2} & \text { if } y \in \partial \Omega, \\ 0 & \text { if } y \in \mathbb{R}^{N} \backslash \bar{\Omega} .\end{cases}
$$

Proposition 2.10. If $h$ is continuous on $\partial \Omega$ and $y_{0} \in \partial \Omega$, then

$$
\lim _{\Omega \ni y \rightarrow y_{0}} \overline{\bar{u}}_{h}(y)=\frac{1}{2} h\left(y_{0}\right)+\overline{\bar{u}}_{h}\left(y_{0}\right), \quad \lim _{\mathbb{R}^{N} \backslash \bar{\Omega}_{\ni y \rightarrow y_{0}}} \overline{\bar{u}}_{h}(y)=-\frac{1}{2} h\left(y_{0}\right)+\overline{\bar{u}}_{h}\left(y_{0}\right) .
$$

Remark 2.11. If $h \in C(\partial \Omega)$, then $\overline{\bar{u}}_{h} \in C(\partial \Omega) \cap L^{m}(\Omega)$, for each $1 \leq m \leq \infty$.

Indeed, by Propositions 2.8 and 2.10, the function $\varphi: \bar{\Omega} \rightarrow \mathbb{R}$ defined by $\varphi(y)=\overline{\bar{u}}_{h}(y)$ for $y \in \Omega$ and $\varphi\left(y_{0}\right)=(1 / 2) h\left(y_{0}\right)+\overline{\bar{u}}_{h}\left(y_{0}\right)$ for $y_{0} \in \partial \Omega$ is continuous on $\bar{\Omega}$. It follows that $\overline{\bar{u}}_{h} \in C(\partial \Omega)$ and $\varphi \in L^{\infty}(\Omega)$. But $\varphi \equiv \overline{\bar{u}}_{h}$ on $\Omega$ so that $\overline{\bar{u}}_{h} \in L^{\infty}(\Omega)$. Thus, for each $1 \leq m<\infty$, we have

$$
\int_{\Omega}\left|\overline{\bar{u}}_{h}\right|^{m} d x \leq\left\|\overline{\bar{u}}_{h}\right\|_{L^{\infty}(\Omega)}^{m}|\Omega|<\infty,
$$

which shows that $\overline{\bar{u}}_{h} \in L^{m}(\Omega)$. 
Definition 2.12. A Lipschitz domain (or domain with Lipschitz boundary) is a domain in $\mathbb{R}^{N}$ whose boundary can be locally represented as the graph of a Lipschitz continuous function.

Many of the Sobolev embedding theorems require that the domain of study be a Lipschitz domain. All smooth and many piecewise smooth boundaries are Lipschitz boundaries.

Proposition 2.13 (see [2, Theorem 7.26]). Let $\omega$ be a Lipschitz domain in $\mathbb{R}^{N}$. If $N<p<\infty$, then $W^{1, p}(\omega)$ is continuously embedded in $C^{0, \alpha}(\bar{\omega})$ with $\alpha=1-N / p$.

Proposition 2.14 (see [2, page 155]). If $\omega$ is a Lipschitz domain, then $C^{\infty}(\bar{\omega})$ is dense in $W^{1, p}(\omega)$ for $1 \leq p<\infty$.

\section{Proof of Theorem 1.3}

Since $\Omega$ is bounded, we can assume without loss of generality that $p<\infty$.

Proof of (a). Suppose that $p>N$. Then, $f \in C^{0, \alpha}(\bar{\Omega})$ with $\alpha=1-N / p$ (cf. Proposition 2.13).

Proof of (1.10) when $y \in \Omega$. We define $F: \bar{\Omega} \backslash\{y\} \rightarrow \mathbb{R}^{N}$ as follows:

$$
F(x)=(f(x)-f(y)) \nabla E(x-y)=\frac{f(x)-f(y)}{\omega_{N}|x-y|^{N}}(x-y) .
$$

Note that $F \notin C^{1}(\Omega)$. We overcome this problem by choosing $\epsilon>0$ small enough such that $\bar{B}_{\epsilon}(y)$, respectively, $\bar{B}_{\epsilon}\left(a_{i}\right)\left(a_{i} \in A \backslash\{y\}\right)$, is contained within $\Omega$ and every two such closed balls are disjoint. Therefore, $F \in C^{1}\left(D_{\epsilon}\right) \cap C\left(\bar{D}_{\epsilon}\right)$, where $D_{\epsilon}=\Omega \backslash\left(\bigcup_{i \in I} \bar{B}_{\epsilon}\left(a_{i}\right) \cup \bar{B}_{\epsilon}(y)\right)$.

Using Proposition 2.5, we arrive at

$$
\begin{aligned}
\int_{D_{\epsilon}} \operatorname{div} F d x= & \int_{\partial \Omega}(f(x)-f(y)) \frac{\partial E}{\partial \mathcal{v}}(x-y) d \sigma(x)-\frac{1}{\epsilon^{N-1-\alpha}} \int_{\partial B_{\epsilon}(y)} \frac{f(x)-f(y)}{\omega_{N}|x-y|^{\alpha}} d \sigma(x) \\
& -\frac{1}{\omega_{N}} \sum_{i \in I, a_{i} \neq y} \int_{\partial B_{\epsilon}\left(a_{i}\right)} \frac{f(x)-f(y)}{\epsilon|x-y|^{N}}\left\langle x-y, x-a_{i}\right\rangle d \sigma(x) .
\end{aligned}
$$

We see that

$$
\lim _{\epsilon \rightarrow 0} \frac{1}{\epsilon^{N-1-\alpha}} \int_{\partial B_{\epsilon}(y)} \frac{f(x)-f(y)}{|x-y|^{\alpha}} d \sigma(x)=0 .
$$

Indeed, by Proposition 2.13, there exists a constant $L>0$ such that

$$
\begin{aligned}
0 & \leq \frac{1}{\epsilon^{N-1-\alpha}}\left|\int_{\partial B_{\epsilon}(y)} \frac{f(x)-f(y)}{|x-y|^{\alpha}} d \sigma(x)\right| \\
& \leq \frac{L}{\epsilon^{N-1-\alpha}} \int_{\partial B_{\epsilon}(y)} d \sigma(x)=L \omega_{N} \epsilon^{\alpha} \longrightarrow 0 \quad \text { as } \epsilon \longrightarrow 0 .
\end{aligned}
$$

Notice that, for each $i \in I$ with $a_{i} \neq y$, there exists a constant $C_{i}>0$ such that

$$
|f(x)-f(y)| \leq C_{i}|x-y|^{N-1}, \quad \forall x \in \bar{B}_{\epsilon}\left(a_{i}\right)
$$


(since $\left.y \notin \bar{B}_{\epsilon}\left(a_{i}\right)\right)$. Hence, if $i \in I$ such that $a_{i} \neq y$, then

$$
\left|\int_{\partial B_{\epsilon}\left(a_{i}\right)} \frac{f(x)-f(y)}{\epsilon|x-y|^{N}}\left\langle x-y, x-a_{i}\right\rangle d \sigma(x)\right| \leq \int_{\partial B_{\epsilon}\left(a_{i}\right)} \frac{|f(x)-f(y)|}{|x-y|^{N-1}} d \sigma(x) \leq C_{i} \omega_{N} \epsilon^{N-1} .
$$

By (3.2)-(3.6) and Gauss lemma, it follows that

$$
\begin{aligned}
\lim _{\epsilon \rightarrow 0} \int_{D_{\epsilon}} \operatorname{div} F(x) d x & =\int_{\partial \Omega}(f(x)-f(y)) \frac{\partial E}{\partial \mathcal{V}}(x-y) d \sigma(x) \\
& =\int_{\partial \Omega} f(x) \frac{\partial E}{\partial \mathcal{v}}(x-y) d \sigma(x)-f(y)
\end{aligned}
$$

Recall that $x \mapsto E(x-y)$ is harmonic on $\mathbb{R}^{N} \backslash\{y\}$. Thus, from (3.1), we derive that

$$
\operatorname{div} F(x)=\langle\nabla f(x), \nabla E(x-y)\rangle, \quad \forall x \in D_{\epsilon}
$$

From Lemma 2.2(ii), we know that

$$
y \longmapsto \int_{\Omega}\langle\nabla E(x-y), \nabla f(x)\rangle d x \text { is continuous on } \bar{\Omega} .
$$

From (3.7) and (3.8), we find

$$
\int_{\Omega}\langle\nabla f(x), \nabla E(x-y)\rangle d x=\lim _{\epsilon \rightarrow 0} \int_{D_{\epsilon}} \operatorname{div} F(x) d x=\int_{\partial \Omega} f(x) \frac{\partial E}{\partial v}(x-y) d \sigma(x)-f(y),
$$

which concludes the proof of (1.10) for $y \in \Omega$.

Proof of (1.10) when $y \in \partial \Omega$. We apply (1.10) to get $f(t)$ with $t \in \Omega$. Then, let $t \rightarrow y$. Thus, using (3.9) and the continuity of $f$ on $\bar{\Omega}$, we obtain

$$
f(y)=\lim _{\Omega \ni t \rightarrow y} f(t)=\lim _{\Omega \ni t \rightarrow y} \overline{\bar{u}}_{f}(t)-\int_{\Omega}\langle\nabla E(x-y), \nabla f(x)\rangle d x
$$

From Proposition 2.10, we know that

$$
\lim _{\Omega \ni t \rightarrow y} \overline{\bar{u}}_{f}(t)=\frac{f(y)}{2}+\overline{\bar{u}}_{f}(y)
$$

By combining (3.11) and (3.12), we attain (1.10).

Proof of (b). Assume that $f \in C(\bar{\Omega})$ and $p \geq 1$. Let $y \in \mathbb{R}^{N} \backslash \bar{\Omega}$ be fixed.

We define the vector field $Z: \bar{\Omega} \rightarrow \mathbb{R}^{N}$ by

$$
Z(x)=f(x) \nabla E(x-y)=\frac{f(x)}{\omega_{N}|x-y|^{N}}(x-y), \quad \forall x \in \bar{\Omega} .
$$


Clearly, $Z \in C^{1}(\Omega \backslash A) \cap C(\bar{\Omega})$. Let $\epsilon>0$ be fixed such that $\bar{B}_{\epsilon}\left(a_{i}\right) \subset \Omega$ for every $i \in I$ and $\bar{B}_{\epsilon}\left(a_{i}\right) \cap$ $\bar{B}_{\epsilon}\left(a_{j}\right)=\varnothing$ for all $i, j \in I$ with $i \neq j$. Set $\Omega_{\epsilon}:=\Omega \backslash\left(\bigcup_{i \in I} \bar{B}_{\epsilon}\left(a_{i}\right)\right)$. By applying Proposition 2.5 to $Z: \Omega_{\epsilon} \rightarrow \mathbb{R}^{N}$, we obtain

$$
\int_{\Omega_{\epsilon}} \operatorname{div} Z(x) d x=\int_{\partial \Omega} f(x) \frac{\partial E}{\partial v}(x-y) d \sigma(x)-\frac{1}{\omega_{N}} \sum_{i \in I} \int_{\partial B_{\epsilon}\left(a_{i}\right)} \frac{f(x)\left\langle x-y, x-a_{i}\right\rangle}{\epsilon|x-y|^{N}} d \sigma(x) .
$$

If $M_{i}=\operatorname{dist}\left(y, \bar{B}_{\epsilon}\left(a_{i}\right)\right)$, then $M_{i}>0$ for every $i \in I$ (since $\left.y \notin \bar{\Omega}\right)$. Hence, for each $i \in I$,

$$
\left|\int_{\partial B_{\epsilon}\left(a_{i}\right)} \frac{f(x)\left\langle x-y, x-a_{i}\right\rangle}{\epsilon|x-y|^{N}} d \sigma(x)\right| \leq \int_{\partial B_{\epsilon}\left(a_{i}\right)} \frac{|f(x)|}{|x-y|^{N-1}} d \sigma(x) \leq \frac{\|f\|_{L^{\infty}(\Omega)}}{M_{i}^{N-1}} \omega_{N} \epsilon^{N-1} .
$$

By (3.14) and (3.15), it follows that

$$
\lim _{\epsilon \rightarrow 0} \int_{\Omega_{\epsilon}} \operatorname{div} Z(x) d x=\int_{\partial \Omega} f(x) \frac{\partial E}{\partial \mathcal{v}}(x-y) d \sigma(x) .
$$

Note that $x \mapsto|x-y|^{1-N}$ is continuous on $\bar{\Omega}$. By Hölder's inequality, $x \mapsto\langle\nabla f(x), \nabla E(x-y)\rangle$ is integrable on $\Omega$. Since $x \mapsto E(x-y)$ is harmonic on $\mathbb{R}^{N} \backslash\{y\}$, we find

$$
\operatorname{div} Z(x)=\langle\nabla f(x), \nabla E(x-y)\rangle, \quad \forall x \in \Omega_{\epsilon} .
$$

Therefore, using (3.16), we obtain

$$
\int_{\Omega}\langle\nabla f(x), \nabla E(x-y)\rangle d x=\lim _{\epsilon \rightarrow 0} \int_{\Omega_{\varepsilon}} \operatorname{div} Z(x) d x=\int_{\partial \Omega} f(x) \frac{\partial E}{\partial \mathcal{v}}(x-y) d \sigma(x) .
$$

This completes the proof of Theorem 1.3.

\section{Proof of Theorem 1.1}

As before, we can assume that $g \in W^{1, p}(\Omega)$ with $p<\infty$. By Proposition 2.14, there exists a sequence $g_{n} \in C^{\infty}(\bar{\Omega})$ such that $g_{n} \rightarrow g$ in $W^{1, p}(\Omega)$, that is,

$$
\lim _{n \rightarrow \infty}\left\|g_{n}-g\right\|_{L^{p}(\Omega)}=0, \quad \lim _{n \rightarrow \infty}\left\|\frac{\partial g_{n}}{\partial x_{i}}-\frac{\partial g}{\partial x_{i}}\right\|_{L^{p}(\Omega)}=0, \quad \forall i \in\{1,2, \ldots, N\} .
$$

From Lemma 2.1, we know that, up to a subsequence (relabeled $\left(g_{n}\right)$ ),

$$
g_{n} \longrightarrow g \quad \text { a.e. in } \Omega \text {. }
$$

Since $C^{1}(\bar{\Omega}) \subseteq W^{1, q}(\Omega)$ for every $q \geq 1$, we can apply Theorem 1.3 to each $g_{n}$ and obtain

$$
\int_{\partial \Omega} g_{n}(x) \frac{\partial E}{\partial v}(x-y) d \sigma(x)-\int_{\Omega}\left\langle\nabla E(x-y), \nabla g_{n}(x)\right\rangle d x= \begin{cases}g_{n}(y), & \forall y \in \Omega, \\ 0, & \forall y \in \mathbb{R}^{N} \backslash \bar{\Omega} .\end{cases}
$$


Using the definition of $\mathcal{K}_{j}$ in (2.1), we write

$$
\int_{\Omega}\left\langle\nabla E(x-y), \nabla g_{n}(x)\right\rangle d x=\frac{1}{\omega_{N}} \sum_{j=1}^{N} \int_{\Omega} \frac{x_{j}-y_{j}}{|x-y|^{N}} \frac{\partial g_{n}}{\partial x_{j}}(x) d x=\frac{1}{\omega_{N}} \sum_{j=1}^{N} \mathcal{K}_{j}\left(\frac{\partial g_{n}}{\partial x_{j}}\right)(y) .
$$

From (4.1) and Lemma 2.2, it follows that for every $j \in\{1,2, \ldots, N\}$,

$$
\begin{gathered}
\lim _{n \rightarrow \infty}\left\|\mathcal{K}_{j}\left(\frac{\partial g_{n}}{\partial x_{j}}\right)-\mathcal{K}_{j}\left(\frac{\partial g}{\partial x_{j}}\right)\right\|_{L^{p}(\Omega)}=0 \quad \text { if } 1 \leq p \leq N \\
\mathcal{K}_{j}\left(\frac{\partial g_{n}}{\partial x_{j}}\right) \longrightarrow \mathcal{K}_{j}\left(\frac{\partial g}{\partial x_{j}}\right) \text { in } C(\bar{\Omega}) \text { as } n \longrightarrow \infty \text { if } p>N .
\end{gathered}
$$

Hence, passing eventually to a subsequence (denoted again by $\left(g_{n}\right)$ ), we have

$$
\lim _{n \rightarrow \infty} \mathcal{K}_{j}\left(\frac{\partial g_{n}}{\partial x_{j}}\right)(y)=\mathcal{K}_{j}\left(\frac{\partial g}{\partial x_{j}}\right)(y) \quad \text { a.e. } y \in \Omega, \forall j \in\{1,2, \ldots, N\} \text {. }
$$

This, jointly with (4.4), implies that

$$
\lim _{n \rightarrow \infty} \int_{\Omega}\left\langle\nabla E(x-y), \nabla g_{n}(x)\right\rangle d x=\int_{\Omega}\langle\nabla E(x-y), \nabla g(x)\rangle d x \quad \text { a.e. } y \in \Omega \text {. }
$$

Hence, passing to the limit $n \rightarrow \infty$ in (4.3) and using (4.2), we reach (1.8).

Proof of (1.9). Let $y \in \mathbb{R}^{N} \backslash \bar{\Omega}$ be arbitrary. Then, $x \mapsto|x-y|^{1-N}$ is continuous on $\bar{\Omega}$. Let $p^{\prime}$ denote the conjugate exponent to $p$ (i.e., $1 / p+1 / p^{\prime}=1$ ). By Hölder's inequality,

$$
\begin{aligned}
\int_{\Omega}\left|\left\langle\nabla E(x-y), \nabla g_{n}(x)-\nabla g(x)\right\rangle\right| d x \\
\quad \leq \frac{1}{\omega_{N}}\left(\int_{\Omega} \frac{d x}{|x-y|^{(N-1) p^{\prime}}}\right)^{1 / p^{\prime}}\left(\int_{\Omega}\left|\nabla\left(g_{n}-g\right)(x)\right|^{p} d x\right)^{1 / p} .
\end{aligned}
$$

Thus, using (4.1) and Lemma 2.4, we infer that

$$
\lim _{n \rightarrow \infty} \int_{\Omega}\left\langle\nabla E(x-y), \nabla g_{n}(x)\right\rangle d x=\int_{\Omega}\langle\nabla E(x-y), \nabla g(x)\rangle d x, \quad \forall y \in \mathbb{R}^{N} \backslash \bar{\Omega} .
$$

Letting $n \rightarrow \infty$ in (4.3), we conclude (1.9). This finishes the proof of Theorem 1.1.

\section{Other results and applications to inequalities}

If $f:[a, b] \rightarrow \mathbb{R}$ is absolutely continuous on $[a, b]$, then the Montgomery identity holds:

$$
f(x)=\frac{1}{b-a} \int_{a}^{b} f(t) d t+\frac{1}{b-a} \int_{a}^{b} p(t, x) f^{\prime}(t) d t \quad \text { for } x \in[a, b]
$$


where $p:[a, b]^{2} \rightarrow \mathbb{R}$ is given by

$$
p(t, x)= \begin{cases}t-a & \text { if } a \leq t \leq x \\ t-b & \text { if } x<t \leq b\end{cases}
$$

In the last decade, many authors (see, e.g., [6] and the references therein) have extended the above result for different classes of functions defined on a compact interval, including functions of bounded variation, monotonic functions, convex functions, $n$-time differentiable functions whose derivatives are absolutely continuous or satisfy different convexity properties, and so forth, and they pointed out sharp inequalities for the absolute value of the difference

$$
D(f ; x):=f(x)-\frac{1}{b-a} \int_{a}^{b} f(t) d t, \quad x \in[a, b] .
$$

The obtained results have been applied in approximation theory, numerical integration, information theory, and other related domains.

If $f$ is absolutely continuous on $[a, b]$, then we have the following Ostrowski-type inequalities (see, e.g., [6, page 2]):

$$
\begin{aligned}
& |D(f ; x)| \\
& \leq \begin{cases}{\left[\frac{1}{4}+\left(\frac{x-(a+b) / 2}{b-a}\right)^{2}\right](b-a)\left\|f^{\prime}\right\|_{L^{\infty}}} & \text { if } f^{\prime} \in L^{\infty}[a, b], \\
\frac{(b-a)^{1 / p}}{(p+1)^{1 / p}}\left[\left(\frac{x-a}{b-a}\right)^{p+1}+\left(\frac{b-x}{b-a}\right)^{p+1}\right]^{1 / p}\left\|f^{\prime}\right\|_{L^{q}} & \text { if } f^{\prime} \in L^{q}[a, b] \text { with } q>1, \\
{\left[\frac{1}{2}+\left|\frac{x-(a+b) / 2}{b-a}\right|\right]\left\|f^{\prime}\right\|_{L^{1}}} & \end{cases}
\end{aligned}
$$

where $p$ is the conjugate exponent to $q$. The constants $1 / 4,(p+1)^{-1 / p}$, and $1 / 2$ are best possible in the sense that they cannot be replaced by smaller constants.

If the function $f:[a, b] \times[c, d] \rightarrow \mathbb{R}$ has continuous partial derivatives $\partial f(t, s) / \partial t$, $\partial f(t, s) / \partial s$, and $\partial^{2} f(t, s) / \partial t \partial s$ on $[a, b] \times[c, d]$, then one has the representation (see [6, page 307])

$$
\begin{aligned}
f(x, y)=\frac{1}{(b-a)(d-c)}[ & \int_{a}^{b} \int_{c}^{d} f(t, s) d t d s+\int_{a}^{b} \int_{c}^{d} p(t, x) \frac{\partial f(t, s)}{\partial t} d t d s \\
& \left.+\int_{a}^{b} \int_{c}^{d} q(s, y) \frac{\partial f(t, s)}{\partial s} d t d s+\int_{a}^{b} \int_{c}^{d} p(t, x) q(s, y) \frac{\partial^{2} f(t, s)}{\partial t \partial s} d t d s\right],
\end{aligned}
$$

for each $(x, y) \in[a, b] \times[c, d]$, where $p$ is defined by (5.2) and $q$ is the corresponding kernel for the interval $[c, d]$. Another representation for $f:[a, b] \times[c, d] \rightarrow \mathbb{R}$ is

$$
\begin{aligned}
f(x, y)= & \frac{1}{b-a} \int_{a}^{b} f(t, y) d t+\frac{1}{d-c} \int_{c}^{d} f(x, s) d s-\frac{1}{(b-a)(d-c)} \int_{a}^{b} \int_{c}^{d} f(t, s) d t d s \\
& +\frac{1}{(b-a)(d-c)} \int_{a}^{b} \int_{c}^{d} p(t, x) q(s, y) \frac{\partial^{2} f(t, s)}{\partial t \partial s} d t d s,
\end{aligned}
$$


for each $(x, y) \in[a, b] \times[c, d]$, provided $\partial^{2} f(t, s) / \partial t \partial s$ is continuous on $[a, b] \times[c, d]$ (see $[6$, page 294]).

For various Ostrowski-type inequalities, the reader is referred to the book in $[6$, Chapters 5 and 6] and the papers in $[7,8]$.

In this section, we give a representation formula for $f$ in terms of the integral mean value over $\Omega$ (under the same assumptions on $f$ as in Theorem 1.3).

Theorem 5.1. One assumes that $f \in W^{1, p}(\Omega) \cap C^{1}(\Omega \backslash A)$, where $p>N$ and $A=\left(a_{i}\right)_{i \in I}$ is a finite family of points in $\Omega$. The following representation formula holds:

$$
\begin{aligned}
f(y)= & \frac{1}{|\Omega|} \int_{\Omega} f(x) d x+\int_{\partial \Omega}\left(\frac{1}{\omega_{N}|x-y|^{N}}-\frac{1}{N|\Omega|}\right) f(x)\langle x-y, v\rangle d \sigma(x) \\
& -\int_{\Omega}\left(\frac{1}{\omega_{N}|x-y|^{N}}-\frac{1}{N|\Omega|}\right)\langle\nabla f(x), x-y\rangle d x, \quad \forall y \in \Omega .
\end{aligned}
$$

Proof. We prove that

$$
\int_{\Omega} f(x) d x=\frac{1}{N} \int_{\partial \Omega} f(x)\langle x-z, v\rangle d \sigma(x)-\frac{1}{N} \int_{\Omega}\langle\nabla f(x), x-z\rangle d x, \quad \forall z \in \mathbb{R}^{N} .
$$

Let $z \in \mathbb{R}^{N}$ be arbitrary. We define $G: \bar{\Omega} \rightarrow \mathbb{R}^{N}$ by $G(x)=f(x)(x-z)$. Let $\epsilon>0$ be small such that $\bar{B}_{\epsilon}\left(a_{i}\right) \subset \Omega$ for every $i \in I$ and $\bar{B}_{\epsilon}\left(a_{i}\right) \cap \bar{B}_{\epsilon}\left(a_{j}\right)=\varnothing$ for all $i, j \in I$ with $i \neq j$. Set $U_{\epsilon}=\Omega \backslash\left(\bigcup_{i \in I} \bar{B}_{\epsilon}\left(a_{i}\right)\right)$. We have $G \in C^{1}\left(U_{\epsilon}\right) \cap C\left(\bar{U}_{\epsilon}\right)$. By Proposition 2.5, we find

$$
\int_{U_{\epsilon}} \operatorname{div} G(x) d x=\int_{\partial \Omega} f(x)\langle x-z, v\rangle d \sigma(x)-\sum_{i \in I} \int_{\partial B_{\epsilon}\left(a_{i}\right)} \frac{f(x)}{\epsilon}\left\langle x-z, x-a_{i}\right\rangle d \sigma(x) .
$$

For $i \in I$, we choose $C_{i}>0$ large such that $|x-z| \leq C_{i}$, for every $x \in \bar{B}_{\epsilon}\left(a_{i}\right)$. Hence,

$$
\left|\int_{\partial B_{\epsilon}\left(a_{i}\right)} \frac{f(x)}{\epsilon}\left\langle x-z, x-a_{i}\right\rangle d \sigma(x)\right| \leq \int_{\partial B_{\epsilon}\left(a_{i}\right)}|f(x)||x-z| d \sigma(x) \leq C_{i}\|f\|_{L^{\infty}(\Omega)} \omega_{N} \epsilon^{N-1}
$$

which implies that

$$
\lim _{\epsilon \rightarrow 0} \int_{\partial B_{\epsilon}\left(a_{i}\right)} \frac{f(x)}{\epsilon}\left\langle x-z, x-a_{i}\right\rangle d \sigma(x)=0, \quad \forall i \in I
$$

Obviously, $f \in L^{1}(\Omega)$ and $x \mapsto\langle\nabla f(x), x-z\rangle$ is integrable on $\Omega$. Therefore, we have

$$
\lim _{\epsilon \rightarrow 0} \int_{U_{\epsilon}} \operatorname{div} G(x) d x=\int_{\Omega} \operatorname{div} G(x) d x=\int_{\Omega}\langle\nabla f(x), x-z\rangle d x+N \int_{\Omega} f(x) d x .
$$

Passing to the limit $\epsilon \rightarrow 0$ in (5.9), then using (5.11) and (5.12), we reach (5.8).

Using representation (1.10) of $f(y)$ with $y \in \Omega$ and representation (5.8) with $z=y$, we conclude (5.7). 
Remark 5.2. More generally, in the framework of Theorem 5.1, one has

$$
\begin{aligned}
f(y)= & \frac{1}{|\Omega|} \int_{\Omega} f(x) d x+\int_{\partial \Omega}\left(\frac{\langle x-y, v\rangle}{\omega_{N}|x-y|^{N}}-\frac{\langle x-z, v\rangle}{N|\Omega|}\right) f(x) d \sigma(x) \\
& -\int_{\Omega}\left(\frac{\langle\nabla f(x), x-y\rangle}{\omega_{N}|x-y|^{N}}-\frac{\langle\nabla f(x), x-z\rangle}{N|\Omega|}\right) d x, \quad \forall y \in \Omega, \forall z \in \mathbb{R}^{N} .
\end{aligned}
$$

As a consequence of Theorems 1.3 and 5.1, we obtain the following.

Corollary 5.3. Assume that $f \in W^{1, p}(\Omega) \cap C^{1}(\Omega \backslash A)$, where $p>N$ and $A=\left(a_{i}\right)_{i \in I}$ is a finite family of points in $\Omega$. The following hold.

(i) An arbitrary value of $f$ is compared below with the double-layer potential with moment $f$ :

$$
\left|f(y)-\int_{\partial \Omega} f(x) \frac{\partial E}{\partial \mathcal{v}}(x-y) d \sigma(x)\right| \leq \frac{\|\nabla f\|_{L^{p}(\Omega)}}{\omega_{N}}\left(\int_{\Omega} \frac{d x}{|x-y|^{(N-1) p^{\prime}}}\right)^{1 / p^{\prime}}, \quad \forall y \in \Omega
$$

where $p^{\prime}$ denotes the conjugate coefficient of $p$ (i.e., $1 / p+1 / p^{\prime}=1$ ). Moreover, for $y \in \Omega$ fixed, the equality in (5.14) is established for the nontrivial function $f(x)= \pm|x-y|$ if $p=\infty$, respectively, $f(x)= \pm|x-y|^{\beta}$ with $\beta=(p-N) /(p-1)$ if $p \in(N, \infty)$.

(ii) For each $a \in \Omega$ and $R>0$ such that $\bar{B}_{R}(a) \subset \Omega$, one has

$$
\begin{aligned}
f(a) & =\frac{1}{\left|B_{R}(a)\right|} \int_{B_{R}(a)} f(x) d x-\frac{1}{\omega_{N}} \int_{B_{R}(a)}\left(\frac{1}{|x-a|^{N}}-\frac{1}{R^{N}}\right)\langle\nabla f(x), x-a\rangle d x \\
& =\frac{1}{\omega_{N} R^{N-1}} \int_{\partial B_{R}(a)} f(x) d \sigma(x)-\frac{1}{\omega_{N}} \int_{B_{R}(a)} \frac{\langle\nabla f(x), x-a\rangle}{|x-a|^{N}} d x .
\end{aligned}
$$

In addition,

$$
\left|f(a)-\frac{1}{\omega_{N} R^{N-1}} \int_{\partial B_{R}(a)} f(x) d \sigma(x)\right| \leq \omega_{N}^{1 / p^{\prime}-1}\left(\frac{R^{N-(N-1) p^{\prime}}}{N-(N-1) p^{\prime}}\right)^{1 / p^{\prime}}\|\nabla f\|_{L^{p}\left(B_{R}(a)\right)}
$$

where the equality is achieved for $f(x)= \pm|x-a|$ if $p=\infty$ and $f(x)= \pm|x-a|^{(p-N) /(p-1)}$ if $p \in(N, \infty)$.

Proof. (i) From $f \in W^{1, p}(\Omega)$ with $p>N$, we have $(N-1) p^{\prime}<N$ so that the right-hand side of (5.14) is finite (see Lemma 2.4). By (1.10) and Hölder's inequality, we have

$$
\left|f(y)-\overline{\bar{u}}_{f}(y)\right|=\left|\int_{\Omega} \frac{\langle x-y, \nabla f(x)\rangle}{\omega_{N}|x-y|^{N}} d x\right| \leq \frac{\|\nabla f\|_{L^{p}(\Omega)}}{\omega_{N}}\left(\int_{\Omega} \frac{d x}{|x-y|^{(N-1) p^{\prime}}}\right)^{1 / p^{\prime}} .
$$

Let $y \in \Omega$ be fixed. We define $f_{p, y}^{ \pm}: \bar{\Omega} \rightarrow \mathbb{R}$ by $f_{p, y}^{ \pm}(x)= \pm|x-y|$ if $p=\infty$ and $\pm|x-y|^{(p-N) /(p-1)}$ if $p \in(N, \infty)$. Clearly, we have $f_{p, y}^{ \pm} \in C(\bar{\Omega}) \cap C^{1}(\Omega \backslash\{y\})$, and for every $x \in \Omega \backslash\{y\}$,

$$
\nabla f_{p, y}^{ \pm}(x)= \pm \frac{x-y}{|x-y|} \quad \text { if } p=\infty, \quad \pm \frac{p-N}{p-1} \frac{x-y}{|x-y|^{(p+N-2) /(p-1)}} \quad \text { if } p \in(N, \infty) \text {. }
$$


Since $C(\bar{\Omega}) \subset L^{p}(\Omega)$, we infer that $f_{p, y}^{ \pm} \in W^{1, p}(\Omega)$ and

$$
\begin{aligned}
& \left\|\nabla f_{p, y}^{ \pm}(x)\right\|_{L^{p}(\Omega)}=1 \quad\left(\text { resp., } \frac{p-N}{p-1}\left(\int_{\Omega} \frac{d x}{|x-y|^{(N-1) p^{\prime}}}\right)^{1 / p}\right) \\
& \text { if } p=\infty \quad \text { (resp., } p \in(N, \infty)) \text {. }
\end{aligned}
$$

By (1.10) and (5.18), the left-hand side (LHS) of (5.14) for $f_{p, y}^{ \pm}$is

$$
(\mathrm{LHS})=\left|\int_{\Omega} \frac{\left\langle x-y, \nabla f_{p, y}^{ \pm}(x)\right\rangle}{\omega_{N}|x-y|^{N}} d x\right|= \begin{cases}\frac{1}{\omega_{N}} \int_{\Omega} \frac{d x}{|x-y|^{N-1}} & \text { if } p=\infty \\ \frac{p-N}{\omega_{N}(p-1)} \int_{\Omega} \frac{d x}{|x-y|^{(N-1) p^{\prime}}} & \text { if } p \in(N, \infty) .\end{cases}
$$

A simple calculation shows that the right-hand side of (5.14) for $f_{p, y}^{ \pm}$equals the above LHS.

(ii) The first identity of (5.15) follows from Theorem 5.1, while the second follows from Theorem 1.3 (with $\Omega=B_{R}(a)$ and $y=a$ ). Notice that

$$
\int_{B_{R}(a)} \frac{d x}{|x-a|^{(N-1) p^{\prime}}}=\int_{0}^{R}\left(\int_{\partial B_{\rho}(a)} \frac{d \sigma(x)}{|x-a|^{(N-1) p^{\prime}}}\right) d \rho=\frac{\omega_{N} R^{N-(N-1) p^{\prime}}}{N-(N-1) p^{\prime}} .
$$

By applying (5.14) with $y=a$ and $\Omega=B_{R}(a)$, we find (5.16).

Remark 5.4. Corollary 5.3(ii) leads to the mean value theorems for harmonic functions. Indeed, if $f$ is harmonic on $\Omega$, then for every ball $B_{R}(a)$ with $\bar{B}_{R}(a) \subset \Omega$, we have

$$
\int_{B_{R}(a)} \frac{\langle\nabla f(x), x-a\rangle}{|x-a|^{N}} d x=\int_{0}^{R}\left(\int_{\partial B_{\rho}(a)} \frac{\partial f}{\partial \mathcal{v}}(x) d \sigma(x)\right) \frac{d \rho}{\rho^{N-1}}=\int_{0}^{R}\left(\int_{B_{\rho}(a)} \Delta f d x\right) \frac{d \rho}{\rho^{N-1}}=0 .
$$

This, jointly with (5.15), implies that

$$
f(a)=\frac{1}{\omega_{N} R^{N-1}} \int_{\partial B_{R}(a)} f(x) d \sigma(x)=\frac{N}{\omega_{N} R^{N}} \int_{B_{R}(a)} f(x) d x .
$$

\section{Acknowledgment}

The authors thank the referees for the useful comments on the first version of this paper.

\section{References}

[1] G. B. Folland, Introduction to Partial Differential Equations, Princeton University Press, Princeton, NJ, USA, 1976.

[2] D. Gilbarg and N. S. Trudinger, Elliptic Partial Differential Equations of Second Order, Springer-Verlag, New York, NY, USA, 1998.

[3] C. Miranda, Partial Differential Equations of Elliptic Type, vol. 2 of Ergebnisse der Mathematik und ihrer Grenzgebiete, Springer-Verlag, New York, NY, USA, 1970.

[4] H. Brezis, Analyse Fonctionelle, Théory et Applications, Masson, Paris, France, 1992. 
[5] R. A. Adams, Sobolev Spaces. Pure and Applied Mathematics, vol. 65, Academic Press, New York, NY, USA, 1975.

[6] S. S. Dragomir and T. M. Rassias, Eds., Ostrowski Type Inequalities and Applications in Numerical Integration, Kluwer Academic Publishers, Dordrecht, The Netherlands, 2002.

[7] S. S. Dragomir, R. P. Agarwal, and N. S. Barnett, "Inequalities for beta and gamma functions via some classical and new integral inequalities," Journal of Inequalities and Applications, vol. 5, no. 2, pp. 103-165, 2000.

[8] S. S. Dragomir, R. P. Agarwal, and P. Cerone, "On Simpson's inequality and applications," Journal of Inequalities and Applications, vol. 5, no. 6, pp. 533-579, 2000. 